\title{
Arachnid types in the Zoological Museum of the Moscow State University. II. Aranei 1 (Arachnida)
}

\section{Типы паукообразных в Зоологическом музее МГУ. II. Aranei 1 (Arachnida)}

\author{
Kirill G. Mikhailov \\ К.Г. Михайлов
}

Zoological Museum MGU, Bolshaya Nikitskaya Str. 2, Moscow 125009 Russia. E-mail: mikhailov2000@gmail.com
Зоологический музей МГУ, ул. Большая Никитская, 2, Москва 125009 Россия.

KEY WORDS: arachnids, Araneae, spiders, museum collections, types, holotypes, paratypes.

КЛЮЧЕВЫЕ СЛОВА: паукообразные, Araneae, пауки, музейные коллекции, типы, голотипы, паратипы.

ABSTRACT: A catalogue of 32 holotypes, 165 paratypes and two syntypes belonging to 50 spider species in seven families (Agelenidae, Araneidae, Linyphiidae, Liocranidae, Lycosidae, Nesticidae, Salticidae) from the collection of the Zoological Museum of the Moscow State University is provided. Other depositories housing the remaining types of the listed species are given as well.

How to cite this article: Mikhailov K.G. 2018. Arachnid types in the Zoological Museum of the Moscow State University. II. Aranei 1 (Arachnida) // Arthropoda Selecta. Vol.27. No.1. P.95-105. doi: 10.15298/arthsel. 27.1.12

РЕЗЮМЕ: Представлен список 32 голотипов, 165 паратипов и 2 синтипов, относящихся к 50 видам пауков (Aranei). Они принадлежат к 7 семействам (Agelenidae, Araneidae, Linyphiidae, Liocranidae, Lycosidae, Nesticidae, Salticidae) и хранятся в Зоологическом музее МГУ. Также перечислены другие хранилища, в которых размещены другие типы указанных видов.

\section{Introduction}

This paper is the second in the series cataloging the arachnid types deposited in the Zoological Museum of the Moscow State University, starting with the spider type collection, the largest one in the Museum. As indicated earlier [Mikhailov, 2016b], Museum's invertebrate type collections include holo-, para- and syntypes of over 3,000 species, of which some 1,000 species belong to Aranei. The Museum's arachnid collection was founded in the 1860 s and currently contains more than 200,000 arachnid specimens, exclusive of Acari [Mikhailov, 2016a].

Traditionally, at least since the 1930s, the following handwritten documentation has been accepted in the Museum: (1) departmental register (Fig. 1), (2) numerous inventory books on diverse invertebrate groups (see Fig. 2 for Aranei), and (3) type index cards (Fig. 3). Unfortunately, only a small part of this documentation has been digitized to date (see Mikhailov, 2016 b, for further details).

In Moscow, the spider collection is the largest one among all Russian and ex-USSR natural history museums, being even larger than those both of the Zoological Institute, Russian Academy of Sciences in St. Petersburg, and of the Institute of Animal Systematics and Ecology, Siberian Branch, Russian Academy of Sciences in Novosibirsk. In the Museum, the spider type collection (Fig. 4) is kept separately from the main collection of this group (Fig. 5).

The following indices are used for arachnids in the registers of the Zoological Museum of the Moscow State University: Ta - spiders (Aranei, or Araneae), $\mathrm{Tb}$ - scorpions (Scorpiones), Tc — solpugids (Solifugae), Td - mites and ticks (Acari), Tk — harvestmen (Opiliones), $\mathrm{Tl}$ - pseudoscorpions (Pseudoscorpiones), Tm - whip scorpions (Uropygi; currently no types).

Altogether, the types of 50 species representing seven families (Agelenidae, Araneidae, Linyphiidae, Liocranidae, Lycosidae, Nesticidae, Salticidae) are listed below, including 32 holotypes, 165 paratypes and two syntypes; 96 accession numbers have been allocated. The Museum card index of the spider type collection is arranged alphabetically by genus name as originally described: Acantholycosa F. Dahl, 1908, Aelurillus Simon, 1884, Agyneta Hull, 1911, etc. The following list of types presents only species belonging to the genera whose names start from 'A'.

\section{Methods}

The following sequence of data is accepted in the type catalogue: species name in the original description; a reference to the first description; transcription of data labels; list of other collections where the re- 

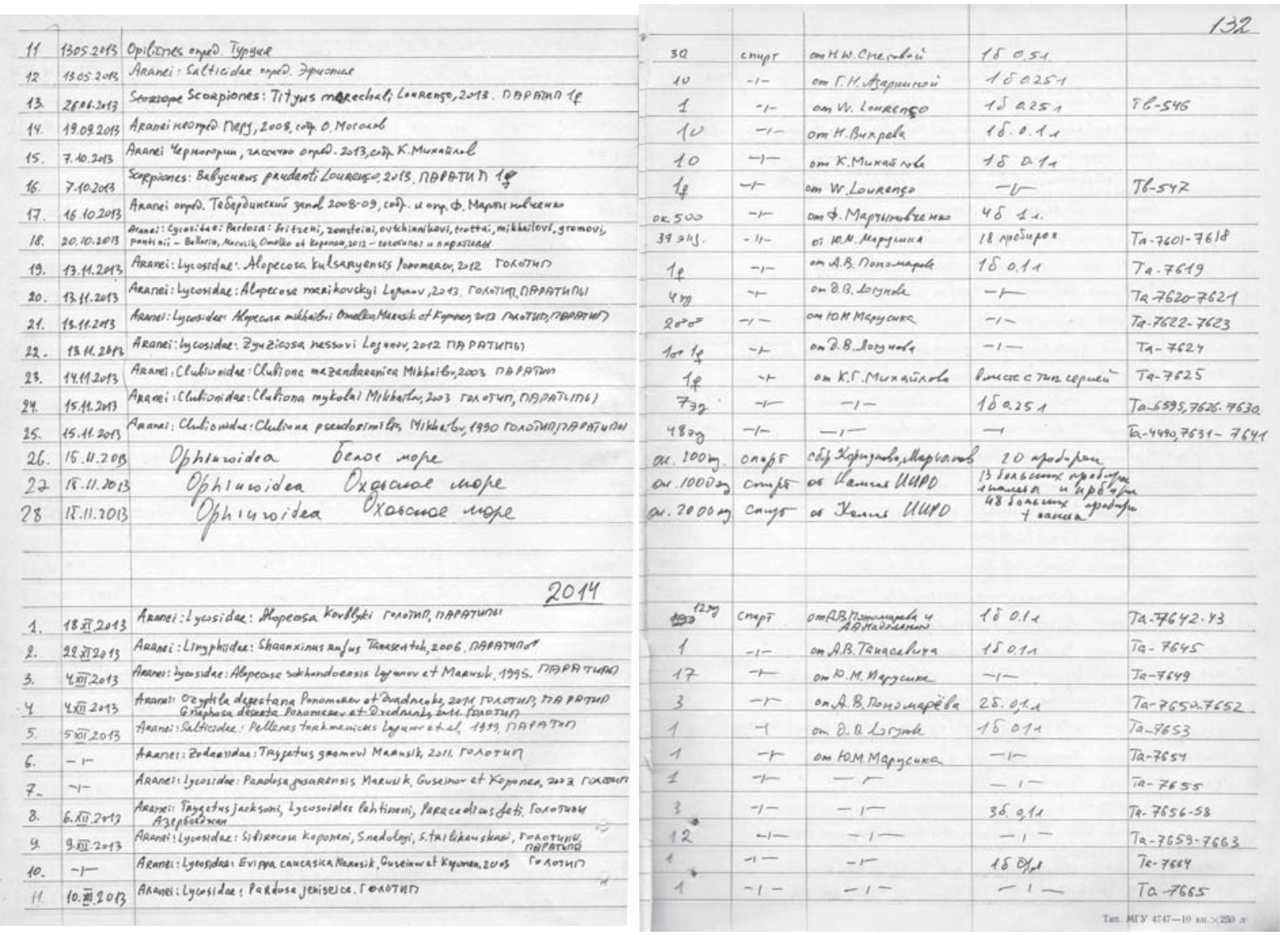

Fig. 1. An acquisition book of the ZMMU Invertebrate Department.

Рис. 1. Книга поступлений отдела беспозвоночных Зоологического музея МГУ.

maining types of the respective species are kept; remarks; current taxonomic status of the species.

When necessary, label abbreviations are put in angled brackets, $<>$. Author's comments to the labels are given in square brackets, []. An English translation of the original Russian labels is provided in figure brackets, \{\} .

Museums or personal collections are abbreviated as follows: ISEA - Institute of Animal Systematics and Ecology, Siberian Branch, Russian Academy of Sciences (Novosibirsk, Russia, curator G.N. Azarkina); JW - personal collection of Jörg Wunderlich (Germany); MMUE — Manchester Museum, University of Manchester (UK, curator D.V. Logunov); NHRS Naturhistoriska Riksmuseet, Stockholm (Sweden, curator G. Lindberg); PSU - Department of Invertebrate Zoology, Perm State University (Russia, curator S.L. Esyunin); SMF — Senckenberg Museum (Frankfurt am Main, Germany, curator P. Jäger); YM - personal collection of Yuri M. Marusik (Magadan, Russia and Turku, Finland); ZIN - Zoological Institute, Russian Academy of Sciences (St. Petersburg, Russia, curator V.A. Krivokhatky); ZMMU — Zoological Museum, Moscow State University (Moscow, Russia, curator K.G. Mikhailov); ZMUT - Zoological Museum, University of Turku (Finland, curator S. Koponen). Where- abouts of other types, except those that are deposited in the ZMMU, is given on the basis of original descriptions. In the ZMMU, museum accession numbers for Aranei are indexed with 'Ta' which is put at the beginning of museum labels. Labels are transcribed literally, with mistakes being indicated in comments.

The following translations of the administrative units are used hereinafter: Area for "область, oblast", District for "район, rayon", Province for "край, kray". The nomenclature follows WSC [2018].

\section{Type catalogue}

aborigenica Zyuzin et Marusik, 1988, Acantholy$\cos a$

Zyuzin, Marusik, 1988: 1083-1084, figs.

Ta-7342. Paratype + , Приморье, пос. Хасан \{Primorie, Khasan\}, 20.VIII.1981, А.К. Панютин \{А.K. Panyutin\}.

The holotype and other paratypes are kept in ZIN

In the original label, the collector name is misspelled as "Пантюлин \{Pantyulin\}".

Current status. Acantholycosa aborigenica Zyuzin et Marusik, 1988 (Lycosidae). gus)

affinisoides Tanasevitch, 1984, Agyneta (AprolaTanasevitch, 1984: 49-50, рис.3 (А-Ж). 


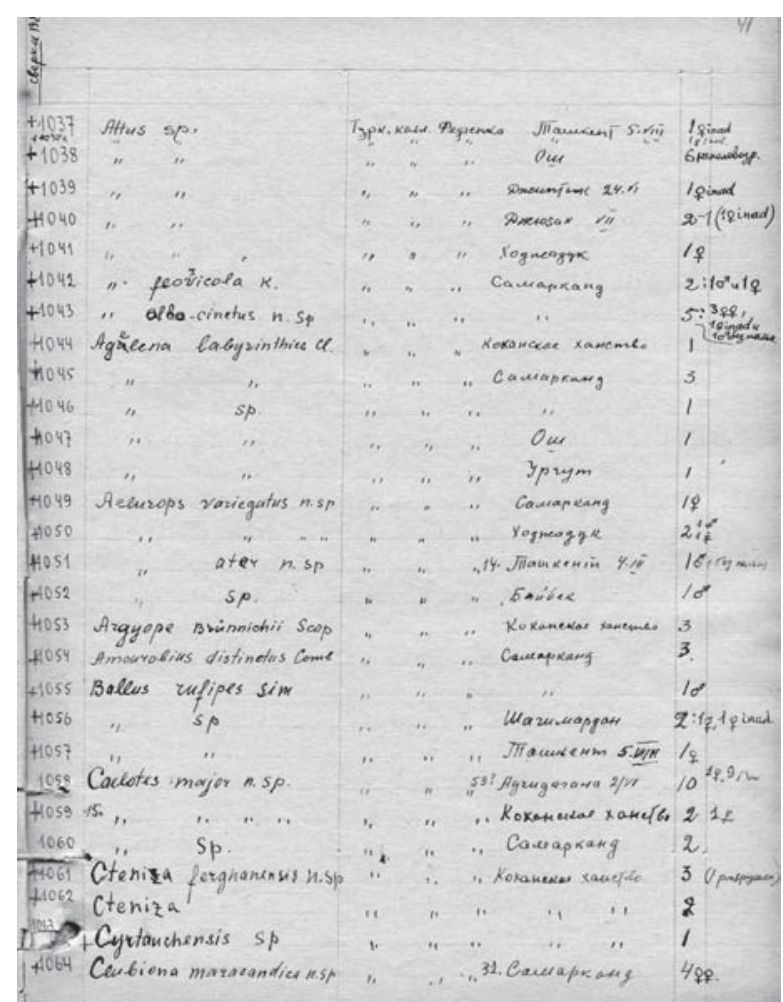

Fig. 2. ZMMU registers for Aranei: above - the old register (1931-1984), below - the new one (2009-).

Рис. 2. Инвентарные книги пауков (Aranei) Зоологического музея МГУ: сверху — старая книга (1931-1984), снизу — новая книга (с 2009 г.).

\begin{tabular}{|c|c|c|}
\hline 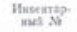 & Massasme nacosas & Mecto colopa \\
\hline 7653 & Prllenes turkemenicus Lemarer & 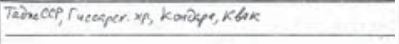 \\
\hline & Macusik of Rabars, 1999 & \\
\hline 7654 & Trygetus gromovi Marusilit, $20 \mathrm{MP}$ & 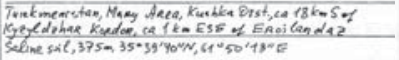 \\
\hline 7655 & Pardose gusarensss Marusiki & 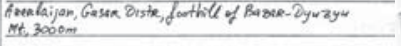 \\
\hline & Gaselnev et koponen, 2003 & \\
\hline 7656 & Tryytas jacksoni Maruske et Gusanow? & 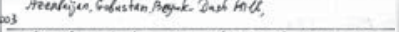 \\
\hline 7657 & 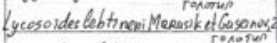 & 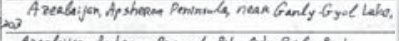 \\
\hline 7658 & 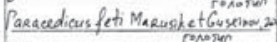 & 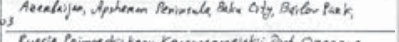 \\
\hline 7859 & 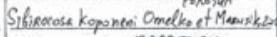 & 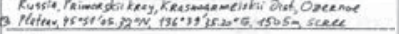 \\
\hline 7660 & Skoponeni RAPATwOA & -+ \\
\hline 7661 & 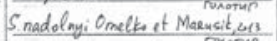 & 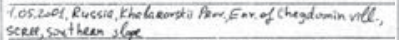 \\
\hline 7662 & 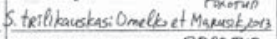 & 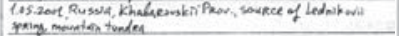 \\
\hline 7663 & S.trilikauskasi nAPATun & $-r$ \\
\hline 7664 & Erippe cancasica Mesusik, Guseriner & 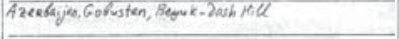 \\
\hline & et Koponen, 2003 & \\
\hline 7665 & Pardosa jenisnica Mareusk in Eskow? & 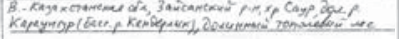 \\
\hline & et Marasik, 1995 & \\
\hline 7666 & & 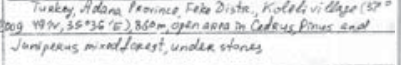 \\
\hline 7667 & T.miceab & $-1-$ \\
\hline 7668 & 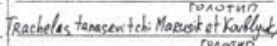 & 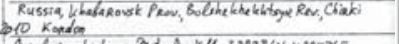 \\
\hline 7669 & Philodromus, ent theer Muster 2009 & 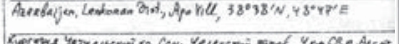 \\
\hline 7670 & Pholous arkit Huler. 20 ronotan? & 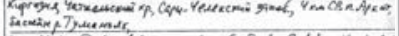 \\
\hline 7671 & Pholens kamkaly thuter, $2 \mathrm{~m}^{\text {ramm }}$ & 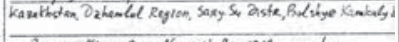 \\
\hline 7672 & 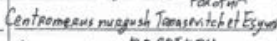 & 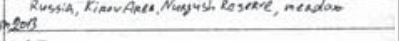 \\
\hline 7673 & C. nuegensh & $-1-$ \\
\hline 7674 & Churgash & \\
\hline 7675 & Helorates altaicus Tanaserstich, 2013 & 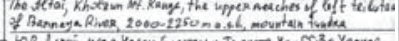 \\
\hline 7676 & 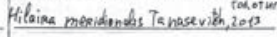 & 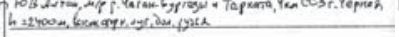 \\
\hline
\end{tabular}

Ta-4197. Holotype $\sigma^{7}$, Эвенкия, р. Кочечум (правый приток <p.> Нижней Тунгуски), 40 км выше устья, лиственничная тайга, подстилка \{Evenkiya, Kochechum River (right tributary of Nizhnaya Tunguska River, $40 \mathrm{~km}$ upstream of the mouth, larch forest, litter\}, 3.VIII.1979, A.A. Вахрушев \{A.A. Vakhrushev\}.

Other types are kept in ZIN.

Current status. Agyneta affinisoides Tanasevitch, 1984 (Linyphiidae).

agrestis Ponomarev, 2007, Agroeca

Ponomarev, 2007: 94-95, fig. 37.

Ta-6880. Holotype 9 , Казахстан, Атырауская обл., пос. Кульсары, в квартире \{Kazakhstan, Atyrau Area, Kulsary, indoor\}, 12.05.1986, В. Романов \{V. Romanov\}.

One paratype ( $(+)$ is not yet transferred to ZMMU.

Current status. Agroeca agrestis Ponomarev, 2007 (Liocranidae).

altaiensis Marusik, Azarkina et Koponen, 2004, Acantholycosa

Marusik et al., 2004: 105, figs 1-9, 56, map 1.

Ta-7538. Paratype + , Altai Province, Charyshskoe Distr., Tigirek Mt. Range, Uba Pass, kurums [=scree], $50^{\circ} 53^{\prime} \mathrm{N}$, $83^{\circ} 48^{\prime} \mathrm{E}, 27.07 .1999$, G. Azarkina et D. Ryzhkov.

The holotype and other paratypes are kept in ISEA.

Current status. Acantholycosa altaiensis Marusik, Azarkina et Koponen, 2004 (Lycosidae).

ater Kroneberg, 1875, Aelurops

Kroneberg, 1875: 50-51, tab. V, fig. 38.

Ta-1051. Holotype ơ [without palps], Туркестанская Ученая Экспедиция Императорского Общества Люби-

21

\begin{tabular}{|c|c|c|c|c|c|c|c|}
\hline 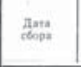 & 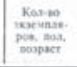 & $\begin{array}{l}\text { Xepaxteg } \\
\text { aperapion }\end{array}$ & 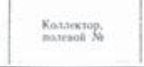 & 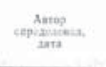 & 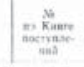 & $\operatorname{Ins}$. & Mppuczenser \\
\hline $10 \overline{2}+19362$ & $10^{7}$ & & E. Anopede & & 5.2014 & 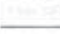 & $x_{2}^{2}$ \\
\hline & + & & 30 & & 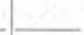 & +8 & $t^{\prime}$ \\
\hline 7042002 & $1 p$ & & dedirgroan & & 6.2014 & & \\
\hline & & & & & & 파달 & 45 \\
\hline 09052001 . & $1 f$ & & by EFGasein & & $7-2014$ & & \\
\hline & & & & & & 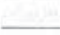 & + \\
\hline 170s:2001: & $1 f$ & $1+4$ & GeRR Jackson & 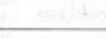 & $8 \cdot 2014$ & $t=$ & 2 \\
\hline 111994 & 17 & & fos EFG Guscinat & & -1 & & \\
\hline (10.1997) & 17 & & -1 & & $-1-$ & 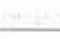 & 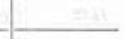 \\
\hline $20 \times 52 \mathrm{~m}$ & $10^{\circ}$ & & MM. Omalt) & & $9-2014$ & +2 & 3825 \\
\hline-1 & $550-3 y$ & & $-1-$ & & $-1-$ & + & +2 \\
\hline Len & $10^{\circ}$ & & (L.Telitikaskas) & - & $-1-$ & $2+$ & thents \\
\hline - & 100 & & $-1-$ & & -1, & & 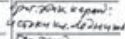 \\
\hline+ & $1 f$ & &,-- & & 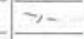 & $3=$ & $x \rightarrow m-4$ \\
\hline serv2001. & 107 & & CyEFGusainor & & $10-2014$ & 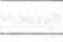 & 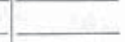 \\
\hline & & & & & & 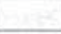 & 782 \\
\hline 22306,1900 & 100 & & KWE Easer & & 11.2014 & 5 & 24 \\
\hline- & & & & $(4)$ & + & 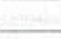 & + \\
\hline 12052009 & 10 & & OSeyyar & & 12.2014 & 80 & by \\
\hline & & & +2 & & & 2 & 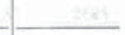 \\
\hline 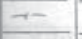 & 107 & & $-1-$ & & $-1-$ & & \\
\hline$x-n z$ zoy & 19 & & besirtanasewtech & & $13-2014$ & $x_{1}^{2}$ & 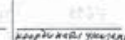 \\
\hline $205 \operatorname{sen} 3$ & $1_{f}$ & & LeN Snegenarye & & 172.14 & 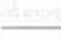 & 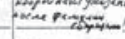 \\
\hline $20 \times \mathrm{CP}_{2}$ & 107 & & 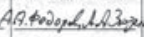 & & $10-2012$ & & 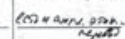 \\
\hline $2 x+19 y$ & 10 & & estzzrrm & & $-1-$ & 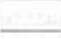 & \\
\hline peanzeas & 10 & & y. LTselisachcheva & & -15.2014 & & \\
\hline$+F$ & $40 \div 2 f f$ & & -1 & & -1 & & - \\
\hline acapod & $10^{\prime}$ & & $-1-$ & & $-1-$ & & \\
\hline Emings. & $15^{\prime}$ & & As Randko leg. & & -1 & & 28 \\
\hline sesen. & If & & AotRoudko ley. & & $-1-$ & + & 30 \\
\hline
\end{tabular}




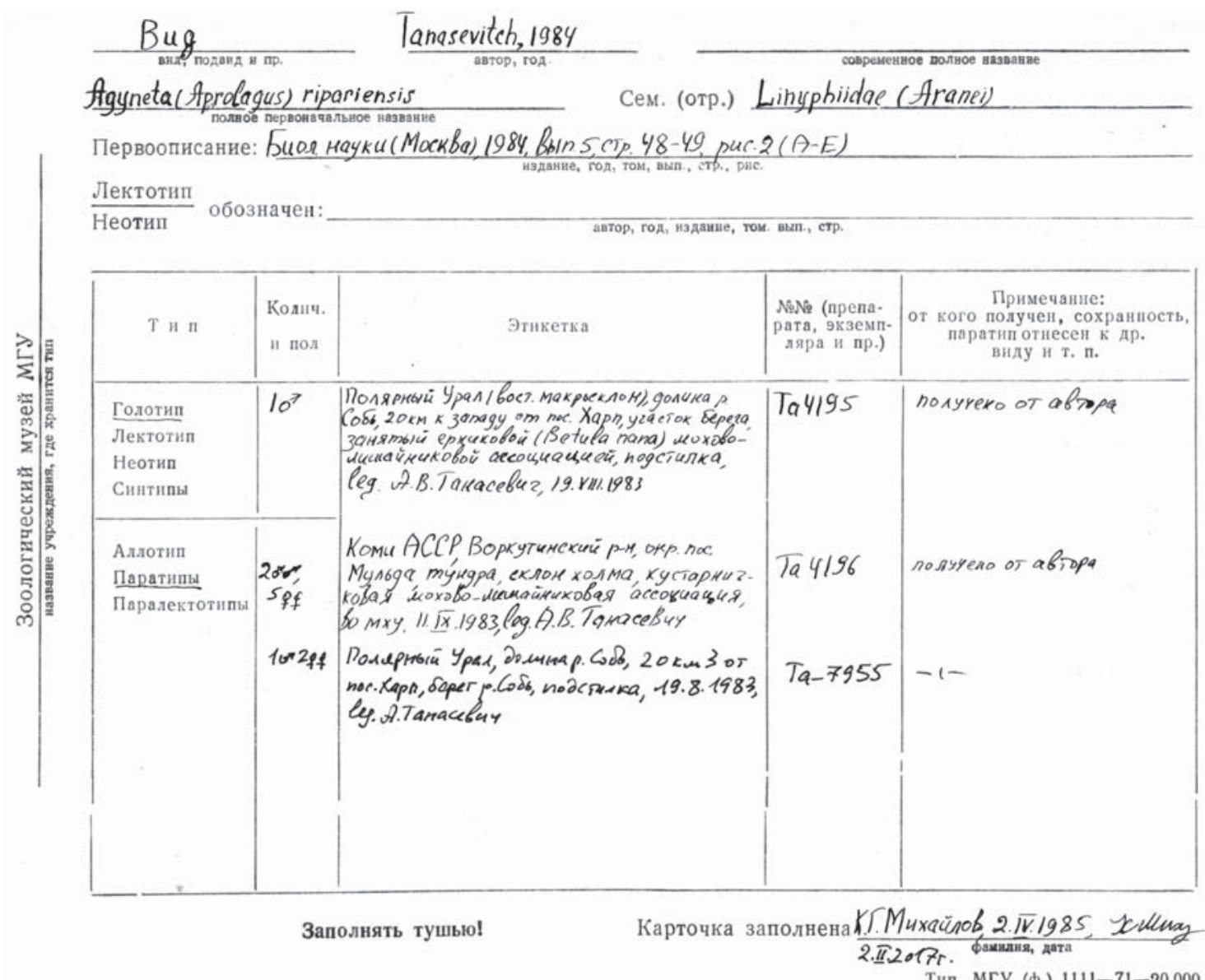

17 FHMEบA H

Тип переописан:

Вид отнесен к другому роду:

другие типы хранятся:

Паратипы посланы в обмен:

Тип исследовали: 


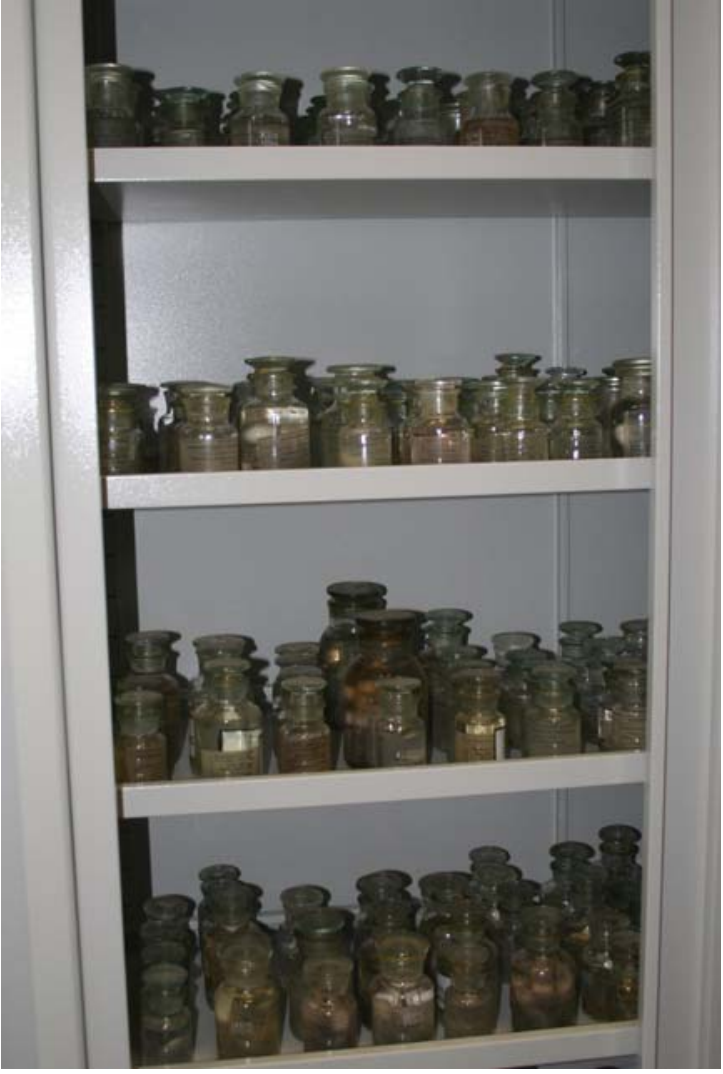

Fig. 4. The type collection of Aranei in ZMMU. Photo by A.V. Sysoev.

Рис. 4. Типовая коллекция пауков (Aranei) Зоологического музея МГУ. Фото А.В. Сысоева.

телей Естествознания. $<$ А.П.> Федченко \{Turkestan Scientific Expedition by the Imperator Society for Friends of Natural Sciences. A.P. Fedchenko\}. 14. Ташкент \{Tashkent\}. 4.III.

The species was described from the holotype only.

Current status. Aelurillus ater (Kroneberg, 1875) (Salticidae).

azarkinae Marusik et Omelko, 2011, Acantholycosa Marusik, Omelko, 2011: 2-5, figs 1-5, 8-12, 26-28.

Ta-7714. Holotype $\sigma^{\top}$, Russia, Maritime Prov., Lazovskii Distr., Sestra Mt., $43^{\circ} 31^{\prime} 52.23^{\prime \prime} \mathrm{N}, 134^{\circ} 02^{\prime} 49.44^{\prime \prime} \mathrm{E}$, scree, 20.06.2005, M.M. Omelko.

Ta-7715. Paratype 9 , Russia, Maritime Prov., Lazovskii Distr., Sestra Mt., $43^{\circ} 31^{\prime} 52.23^{\prime \prime} \mathrm{N}, 134^{\circ} 02^{\prime} 49.44^{\prime \prime} \mathrm{E}$, scree, 20.06.2005, M.M. Omelko.

Other types are kept in the collection of Gornotaezhnaya Station (Ussuriysk Distr., Maritime Prov.).

Current status. Acantholycosa azarkinae Marusik et Omelko, 2011 (Lycosidae).

\section{azerbajdzanicus Dunin, 1984, Aelurillus}

Dunin, 1984: 51, fig. 11

Ta-6790. Holotype + , Azerbaijan, Baku, Zykh, 8.06.1979, coll. P.M. Dunin.

Ta-6791. Paratype + , Azerbaijan, Baku, Zykh, 8.06.1979, coll. P.M. Dunin.

Ta-6792. Paratype $\bigcirc$, Azerbaijan, Apsheron Peninsula, Dyubendy, 21.05.1977, coll. P.M. Dunin.

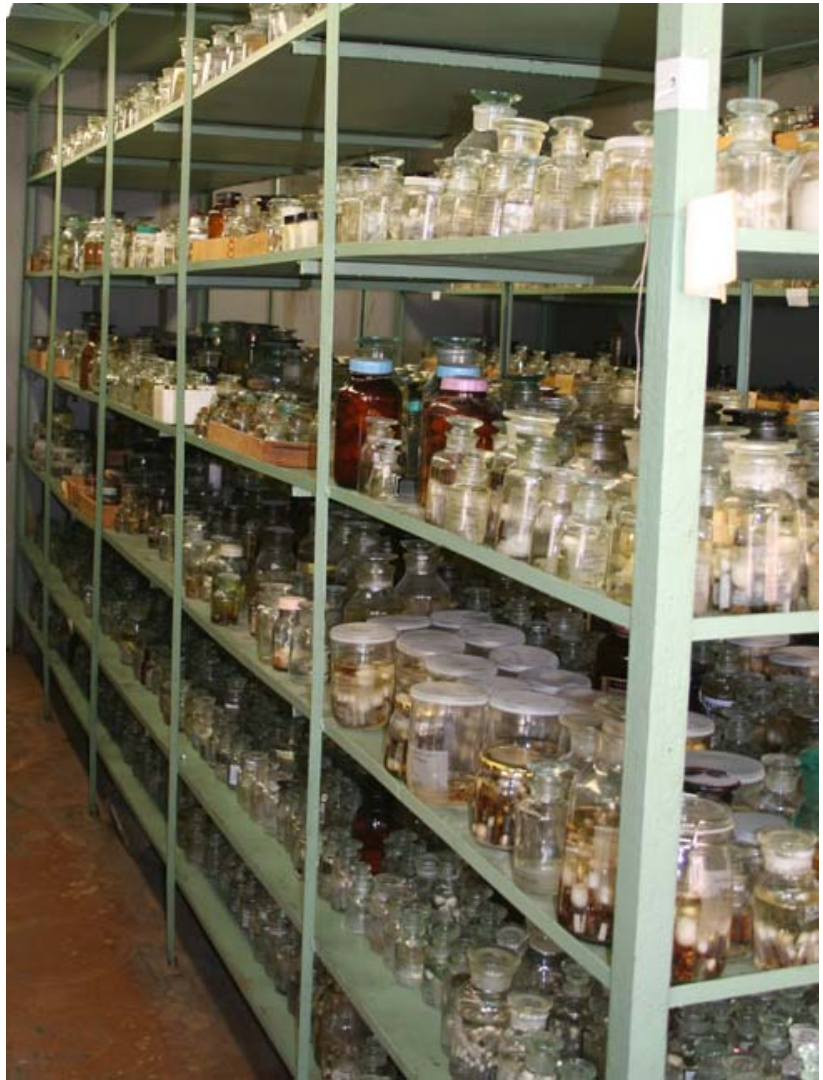

Fig. 5. The main collection of Aranei in ZMMU. Photo by A.V. Sysoev.

Рис. 5. Основная коллекция пауков (Aranei) Зоологического музея МГУ. Фото А.В. Сысоева.

Types are kept in ZMMU only.

In the original description, the misspelled name 'azerbajdzanica' is used in figure captions and a general species list.

Current status. Synonymized with Aelurillus concolor Kulczyński, 1901 (Salticidae) [Azarkina, 2003, initially with A. muganicus Dunin, 1984; see Azarkina, Mirshamsi, 2014 for further details].

brutus Wesołowska, 1996, Aelurillus

Wesołowska, 1996: 23-24, figs 4A, B.

Ta-7501. Holotype + , USSR, Turkmenia, South Ustyurt, Kaplankyrsky Reserve, 2.04. $<19>85$, leg. L.A. Mitroshina.

The species was described from the holotype only.

Current status. Aelurillus brutus Wesołowska, 1996 (Salticidae).

bulavintsevi Tanasevitch, 2016, Agyneta

Tanasevitch, 2016: 75, figs 1-8.

Ta-7750. Holotype $\sigma^{7}$, Якутия, окр. пос. Тикси \{Yakutia, nr. Tiksi\}, VIII.1985, leg. BulavintsevV. $<$ I.>

The species was described from the holotype only.

Current status. Agyneta bulavintsevi Tanasevitch, 2016 (Linyphiidae).

caucasica Guseinov, Marusik et Koponen, 2005, Agelescape

Guseinov et al., 2005: 157, figs 9-12, 69-71, 105.

Ta-7506. Holotype $\$$, CN Azerbaijan, Ismailly Distr., env. of Khanaya Vill., 700 m, 8.07.2001, E.F. Guseinov. 
Ta-7507. Paratypes $2 \circ \circ$, CN Azerbaijan, Ismailly Distr., env. of Khanaya Vill., 700 m, 1.10.2003, E.F. Guseinov. Types are kept in ZMMU only.

Current status. Agelescape caucasica Guseinov, Marusik et Koponen, 2005 (Agelenidae).

cuneata Tanasevitch, 2014, Agyneta

Tanasevitch, 2014: 77, figs 1-9.

Ta-7696. Holotype O', Амурская обл., Селемджинский p-н, о-в на р. Бысса, 2 км выше устья руч. Кукуя \{Amur Area, Selemdzhinsk Distr., an island on Byssa River, $2 \mathrm{~km}$ upstream of Kukuy Creek's mouth\}, 6.VI.2007, E. $<$ M.> Веселова, А. $<$ Б.> Рывкин \{E.M. Veselova, А.В. Ryvkin\}.

The species was described from the holotype only.

Current status. Agyneta cuneata Tanasevitch, 2014 (Linyphiidae).

dubatolovi Azarkina, 2003, Aelurillus

Azarkina, 2003: 95-97, figs 29-50, Table 1.

Ta-6586. Paratypes $50^{7} \sigma^{7}, 1$, Северный Таджикистан, хр. Моголтау \{northern Tadzhikistan, Mogoltau Mt. Range\}, подгорная равнина \{foothill plain\}, 27.02.1983, coll. А.Б. Ненилин \{A.B. Nenilin\}.

Ta-6587. Paratypes $2 \sigma^{7} \sigma^{7}, 1$ क, Туркмения, Ц<ентр>. Копетдаг, 20 км Ю Геок-Тепе, гора Душак \{Turkmenia, Central Kopetdagh, ca $20 \mathrm{~km} \mathrm{~S}$ of Geok-Tepe, Dushak Mt.\}, 2300 м $\{\mathrm{m}\}$, юж. склон $\{\mathrm{S}$ slope $\}$, арчёвое редколесье \{sparse Juniperus stand\}, 20.04.1989, coll. К.Г. Михайлов $\{$ K.G. Mikhailov\}.

Ta-6588. Paratype + , Uzbekistan, Kuraminskii Mt. Range, Bash-Kul, 12.04.1985, coll. S.V. Ovchinnikov.

Ta-6589. Paratype +, Казахстан, Джезказганская обл., Балхашск. р-н, пос. Хасхабье \{Kazakhstan, Dzhezkazghan Area, Balkhash Distr., Khaskhabye\}, VI.1988, О. Ильченко \{O. Ilchenko\}.

Other types are kept in ISEA (holotype, paratypes), MMUE and NHRS.

Current status. Aelurillus dubatolovi Azarkina, 2003 (Salticidae).

dudkorum Marusik, Azarkina et Koponen, 2004,

\section{Acantholycosa}

Marusik et al., 2004: 113-115, figs 59, 73-79, 84-85, map 1.

Ta-7539. Holotype $\sigma^{7}$, Altai, $50 \mathrm{~km} \mathrm{~W}$ of Kosh-Agach, ca. 20-25 km W of Bel'tir, Taltura (Chagan-Uzun) River canyon, 2900-3100 m, mountain stone steppe, 26-28.07. 1999, V.V. Glupov.

Ta-7540. Paratypes 3 우, Altai, $50 \mathrm{~km} \mathrm{~W}$ of KoshAgach, ca. $20-25 \mathrm{~km}$ W of Bel'tir, Taltura (Chagan-Uzun) River canyon, 2900-3100 m, mountain stone steppe, 2628.07.1999, V.V. Glupov.

Other types are kept in ISEA and ZMUT.

Current status. Acantholycosa dudkorum Marusik, Azarkina et Koponen, 2004 (Lycosidae).

\section{lescape}

dunini Guseinov, Marusik et Koponen, 2005, Age-

Guseinov et al., 2005: 158, figs 1, 2, 6-8.

Ta-7508. Holotype $\bigcirc^{7}$, CW Azerbaijan, Khojavend (=Gadrut) Distr., env. of Azykh Vill., 12.07.1977, P.M. Dunin.

Ta-7509. Paratype + , CW Azerbaijan, Khojavend ( $=\mathrm{Ga}-$ drut) Distr., env. of Azykh Vill., 12.07.1977, P.M. Dunin.

Types are kept in ZMMU only.

Current status. Agelescape dunini Guseinov, Marusik et Koponen, 2005 (Agelenidae). helvenacius Logunov, 1993, Aelurillus

Logunov, 1993: 47-49, fig. 1a,b.

Ta-4779. Holotype + , Монголия, Эхийн-Гол \{Mongolia, Ekhiin-Gol\}, оазис \{oasis\}, сухой чий \{dry Achnatherum\}, 30.VII.1983, собр. Н.<Б.> Ананьева \{coll. N.B. Ananieva $\}$.

The species was described from the holotype alone.

Current status. Aelurillus helvenacius Logunov, 1993 (Salticidae).

khakassica Marusik, Azarkina et Koponen, 2004,

\section{Acantholycosa}

Marusik et al., 2004: 119, figs 100-103, map 1.

Ta-7541. Paratypes $10 \sigma^{7} \sigma^{7}$, Russia, SW Khakassia, Abakan Mt. Range, Choosek Mt. Range, $20 \mathrm{~km} \mathrm{SSE} \mathrm{of}$ Mrassu, talus, 1600-1800 m, 7-19.07.1999, D. Lomakin.

The holotype and other paratypes are kept in ISEA.

Current status. Acantholycosa khakassica Marusik, Azarkina et Koponen, 2004 (Lycosidae).

\section{eta)}

kopetdaghensis Tanasevitch, 1989, Agyneta (Meio-

Tanasevitch, 1989: 89-91, figs 1-5.

Та-5430. Holotype ऽ, Туркмения, <Юго->Зап. Копетдаг, ущ. Айдере \{Turkmenia, SW Kopetdagh, Aidere Canyon\}, в подстилке грецкого opexa \{Juglans regia, litter\}, 16.II-11.III.1979, собр. В.Я. Фет \{coll. V.Ya. Fet\}.

Ta-5431. Paratypes $2 \sigma^{7} \sigma^{7}$, Туркмения, <Юго-> Зап. Копетдаг, ущ. Айдере \{Turkmenia, SW Kopetdagh, Aidere Canyon , подстилка \{litter\}, 18.III-8.IV.1979, собр. В.Я. Фет \{coll. V.Ya. Fet\}.

Other types are kept in SMF.

Current status. Agyneta kopetdaghensis Tanasevitch, 1989 (Linyphiidae).

kurchumensis Marusik, Azarkina et Koponen, 2004,

\section{Acantholycosa}

Marusik et al., 2004: 119, figs 82-83, map 1.

Ta-6777. Paratype + , Kazakhstan, East-Kazakhstan Area, Kurchum Mt. Range, Kurchum River upper flow, 23.08.1990, V.K. Zinchenko. $\mathrm{SMF}$ ).

Other types are kept in ISEA (holotype) and JW (now in

Current status. Synonymized with Acantholycosa katunensis Marusik, Azarkina et Koponen, 2004 (Lycosidae) [Fomichev et al., 2016].

laimonasi Tanasevitch, 2006, Agyneta

Tanasevitch, 2006: 29-30, figs 1-7.

Ta-7192. Holotype $\sigma^{7}$, Russia, Khabarovsk Province, Verkhnebureinsky District, Bureinsky $\mathrm{N}<$ ature $<\mathrm{R}<$ eserve $>$, upper reaches of Pravaya Bureya River, source of Lednikovyi River (ca. $52^{\circ} 08^{\prime} \mathrm{N}, 134^{\circ} 26^{\prime} \mathrm{E}$ ), montane tundra, 30.VI. 2000, L. $<$ A. $>$ Trilikauskas.

The species was described from the holotype alone.

Current status. Agyneta laimonasi Tanasevitch, 2006 (Linyphiidae)

laniger Logunov et Marusik, 2000, Aelurillus

Logunov, Marusik, 2000: 265, figs 1-6.

Ta-6304. Paratypes $3 \sigma^{\top} \sigma^{\top}, 2$ 우, Kazakhstan, Pavlodar Area, $\sim 1 \mathrm{~km} \mathrm{~W}$ of Ermak (=Aksu), 14-28.06.1998, leg. A.V. Gromov.

Other types are kept in ISEA (holotype, paratypes) and NHRS. 
Current status. Aelurillus laniger Logunov et Marusik, 2000 (Salticidae).

\section{levii Tanasevitch, 1984, Agyneta (Aprolagus)}

Tanasevitch, 1984: 50-51, pис. 4 (A-3).

Ta-4198. Holotype $\sigma^{7}$, Енисей, Мирное \{Enisei, Mirnoe $\}$ (62 $20^{\prime}$ сев.шир. $\left.\{\mathrm{N}\}\right)$, пойменные кустарниковые заросли \{floodplain shrubs\}, 9.VIII.1979, leg. К.Ю. Еськов $\{$ K.Yu. Eskov\}.

Та-4199. Paratypes $3 \sigma^{\top} \sigma^{7}, 10$ 우, Енисей, Мирное \{Enisei, Mirnoe\}, тальники \{willows\}, 9.VIII.1979, leg. К.Ю. Еськов \{K.Yu. Eskov\}.

Ta-4200. Paratype + , Енисей, Мирное \{Enisei, Mirnoe\}, в речных наносах \{in river drifts\}, 5.VIII.1978, leg. К.Ю. Еськов \{K.Yu. Eskov\}.

Ta-4201. Paratypes $2 \sigma^{7} \sigma^{7}$, Енисей, Мирное, левый берег Енисея \{Enisei, Mirnoe, Enisei Rever left bank\}, тальники \{willows\}, 23.V.1978, leg. К.Ю. Еськов \{K.Yu. Eskov\}.

Та-7245. Paratypes $2 \sigma^{\top} \sigma^{7}, 4$ фо, тальники \{willows\}, 9.VIII.1979, К.Ю. Еськов \{K.Yu. Eskov\}.

Types are kept in ZMMU only. idae).

Current status. Agyneta levii Tanasevitch, 1984 (Linyphi-

levinae Marusik, Azarkina et Koponen, 2004, Acan-

\section{tholycosa}

Marusik et al., 2004: 112, figs 61-65, map 1.

Ta-6775. Holotype $\bigcirc^{7}$, Altai, Katun' Reserve, kurums [=scree], traps, 11.07.2000, N.<V.> Levina.

Ta-7542. Paratype $\sigma^{7}$, Atlai, Sredneye Multinskoye Lake, ca. $30 \mathrm{~km}$ SE of Ust-Koksa, 1600-2200 m, 30.07-5.08.1994, S.I. Golovatch, A.B. Ryvkin.

Types are kept in ZMMU only.

Current status. Acantholycosa levinae Marusik, Azarkina et Koponen, 2004 (Lycosidae).

levyi Guseinov, Marusik et Koponen, 2005, Age-

\section{lescape}

Guseinov et al., 2005: 158, figs 3-5.

Ta-7510. Holotype $\sigma^{7}, \mathrm{CN}$ Azerbaijan, Ismailly Distr., env. of Khanaya Vill., 700 m, 06.2002, E.F. Guseinov.

The species was described from the holotype alone.

Current status. Agelescape levyi Guseinov, Marusik et Koponen, 2005 (Agelenidae).

logunovi Marusik, Azarkina et Koponen, 2004, Acantholycosa

Marusik et al., 2004: 131, figs 88-91, 129, 173-180, map 1.

Ta-6770. Paratype $\sigma^{\top}$, Altai, $50 \mathrm{~km} \mathrm{~W}$ of Kosh-Agach, ca. $20-25 \mathrm{~km} \mathrm{~W}$ of Bel'tir, Taltura (Chagan-Uzun) River canyon, 2200-2300 m, stony debris on the upper border of Larix forest, 25-30.06.1999, D. $<$ V. $>$ Logunov.

Ta-6771. Paratype + , Altai, Chiri Lake, 1800-2000 m, 30.07.1997, A. $<$ V.> Tanasevitch.

Other types are kept in ISEA (holotype, paratypes) and ZMUT.

Current status. Acantholycosa logunovi Marusik, Azarkina et Koponen, 2004 (Lycosidae).

marusiki Azarkina, 2002, Aelurillis

Azarkina, 2002: 255-257, figs 44-53.

Ta-6707. Paratype + , Iran, Fars Prov., bridge on GharaAghatch River, $52^{\circ} 13^{\prime} \mathrm{E}, 29^{\circ} 49^{\prime} \mathrm{N}, 24.05 .2000$, Yu. $<$ M. $>$ Marusik.
Ta-6708. Paratype $0^{7}$, Iran, Fars Prov., Haft-barm, 52 $15^{\prime} \mathrm{E}, 29^{\circ} 45^{\prime} \mathrm{N}, 24.05 .2000$, Yu. $<\mathrm{M}$. $>$ Marusik.

Other types are kept in ISEA, MMUE, YM (holotype, now in SMF) and Dept. of Agricultural Zoology, Plant Pests and Diseases Research Institute (Tehran, Iran).

Current status. Aelurillis marusiki Azarkina, 2003 (Salticidae).

metatarsalis Tanasevitch, 2014, Agyneta

Tanasevitch, 2014: 78-79, figs 10-17.

Ta-7697. Holotype $\sigma^{7}$, Респ. Алтай, Чойский p-н, окр. горы Балтырган \{Altai Republic, Choya Distr., environs of Baltyrgan Mt.\}, 2100 м $\{\mathrm{m}\}, 4.8 .2001$, Н.В. Левина $\{$ N.V. Levina\}.

The species was described from the holotype alone.

Current status. Agyneta metatarsalis Tanasevitch, 2014 (Linyphiidae).

\section{minimontanus Azarkina, 2002, Aelurillis}

Azarkina, 2002: 257-258, figs 54-63.

Ta-6709. Paratypes $1 \sigma^{7}, 1$ ㅇ, IN-14 India, Himachal Pradesh, Sissu Vill., $32^{\circ} 28.1^{\prime} \mathrm{N}, 7^{\circ} 07.9^{\prime} \mathrm{E}, 3150-3500 \mathrm{~m}$, 8-10.06.1999, Yu. $<$ M.> Marusik.

Other types are kept in ISEA, MMUE and YM (holotype, now in SMF).

Current status. Aelurillis minimontanus Azarkina, 2003 (Salticidae).

muganicus Dunin, 1984, Aelurillus

Dunin, 1984: 50-51, figs 12, 13.

Ta-6788. Paratype +, Азербайджан, о-в Глиняный, напротив п. Алят \{Azerbaijan, Glinyanyi Is., opposite Alyat Vil.\}, 15.07.1979, сб. П.М. Дунин \{coll. P.M. Dunin\}.

Ta-6789. Paratypes $4 \bigcirc^{7} \sigma^{7}$, Azerbaijan, Apsheron Peninsula, Bina, 3.10.1973, coll. P.M. Dunin.

Whereabouts of other types (holotype $\sigma^{7}$ and paratype ${ }_{+}$) is not known.

In the original description, the misspelled name A. muganica is used in figure captions and a general species list.

Current status. Synonymized with Aelurillus concolor Kulczyński, 1901 (Salticidae) [Azarkina, Mirshamsi, 2014].

nataliae Esyunin et Efimik, 1998, Aituaria

Esyunin, Efimik, 1998: 147, figs 1-2, 4-7.

Та-6232. Holotype $\sigma^{\top}$, Оренбургская область, Кувандыкский p-н, пос. Айтуарка \{Orenburg Area, Kuvandyk Distr., Aituarka\}, в помещении \{indoor\}, IX.1996, собр. H.C. Мазура \{coll. N.S. Mazura\}.

The species was described from the holotype alone.

Current status. Synonymized with Aituaria pontica (Spassky, 1932) (Nesticidae) [Esyunin, 2017].

oligerae Marusik, Azarkina et Koponen, 2004, Acantholycosa

Marusik et al., 2004: 126-128, figs 19-20, 128, 152-161, map 2.

Ta-6772. Holotype $\sigma^{7}$, Russia, Maritime Province, Lazo Reserve, Sukhoi Klyuch site, edge of stony debris, on slope, 22.06.1981, T. $<$ I. $>$ Oliger.

Ta-6773. Paratypes 2 우, Russia, Maritime Province, Lazo Reserve, Sukhoi Klyuch site, edge of stony debris, on slope, 22.06.1981, T. $<$ I.> Oliger.

Ta-6774. Paratype $\sigma^{\top}$, Russia, Maritime Province, Lazo Reserve, Sukhoi Klyuch site, mixed forest, N exposed slope, 23.06.1981, T. $<$ I. $>$ Oliger. 
Other types are kept in JW (now in SMF) and ZMUT.

Current status. Acantholycosa oligerae Marusik, Azarkina et Koponen, 2004 (Lycosidae).

paraplumalis Marusik, Azarkina et Koponen, 2004, Acantholycosa

Marusik et al., 2004: 112, figs 38-43, 49-52, 55, map 1.

Ta-6758. Holotype $\sigma^{7}$, Altai, Choya Distr., Boityrgan Mt., stony mountain tundra, 12.08.2001, N. $<$ V $>$ Levina.

Ta-6759. Paratype + , Altai, Choya Distr., Boityrgan Mt., stony mountain tundra, $12.08 .2001, \mathrm{~N} .<\mathrm{V}$.> Levina.

Ta-6760. Paratypes 2 우, Altai, Choya Distr., Boityrgan Mt., mountain meadow, 13.08.2001, N.<V.> Levina.

Ta-6761. Paratype + , Altai, Choya Distr., Boityrgan Mt., mountain meadow, 13.08.2001, N.<V.> Levina.

Ta-6762. Paratype + , Altai, Choya Distr., Boityrgan Mt., stony debris, 4.08.2001, N. $<$ V. $>$ Levina.

Ta-6763. Paratypes 4 우, Altai, Choya Distr., Boityrgan Mt., stony tundra, 12.08.2001, N. $<$ V. $>$ Levina.

Ta-6764. Paratypes 5 $9+$, Altai, Choya Distr., Boityrgan Mt., mountain meadow, 13.08.2001, N. Levina.

Ta-6765. Paratypes $2+\circ$, Altai, Choya Distr., Boityrgan Mt., stony tundra, 12.08.2001, N. $<$ V. $>$ Levina.

Ta-6766. Paratype $\sigma^{7}$, Altai, Choya Distr., Boityrgan Mt., forest tundra, 14.08.2001, N. $<$ V.> Levina.

Ta-6767. Paratypes $2+\circ$, Altai, Choya Distr., Boityrgan Mt., stony tundra, 12.08.2001, N. $<$ V.> Levina.

Ta-6768. Paratype ${ }_{+}$, Altai, Choya Distr., Boityrgan Mt., stony tundra, 12.08.2001, N.<V. $>$ Levina.

Ta-6769. Paratype ${ }_{+}$, Altai, Choya Distr., Boityrgan Mt., mountain meadow, 13.08.2001, N. $<$ V.> Levina.

Ta-7543. Paratype ${ }_{+}$, Altai, Choya Distr., Boityrgan Mt., forest tundra and mountain tundra, 14.08.2001, N.<V.> Levina.

Other types are kept in JW (now in SMF), MMUE, NHRS, ZMUT.

Current status. Acantholycosa paraplumalis Marusik, Azarkina et Koponen, 2004 (Lycosidae).

petrophila Marusik, Azarkina et Koponen, 2004,

\section{Acantholycosa}

Marusik et al., 2004: 119, figs 104-107, map 1.

Ta-7544. Paratypes $10 \bigcirc^{7} \sigma^{7}$, Russia, Khakassia, Western Sayan, $50 \mathrm{~km}$ SSW of Tashtyp, central part of Khansyn Mt. Range, scree, 1800-2100 m, 11-24.07.2000, D. Lomakin.

The holotype and other paratypes are kept in ISEA.

Current status. Acantholycosa petrophila Marusik, Azarkina et Koponen, 2004 (Lycosidae).

plumalis Marusik, Azarkina et Koponen, 2004, Acantholycosa

Marusik et al., 2004: 109-112, figs 30-37, 44-48, map 1. Ta-6757. Paratypes 3 우, Altai, Teletskoye Lake, Chiri Vil., scree, 30.07.1997, A. $<$ V. $>$ Tanasevitch.

The holotype and other paratypes are kept in ISEA.

Current status. Acantholycosa plumalis Marusik, Azarkina et Koponen, 2004 (Lycosidae).

\section{lagus)}

pseudosaxatilis Tanasevitch, 1984, Agyneta (Apro-

Tanasevitch, 1984: 47-48, рис. 1 (A-3).

Ta-4193. Holotype O7, Эвенкия, p. Кочечум (правый приток Нижней Тунгуски), 40 км выше устья \{Evenkiya, Kochechum River (right tributary of Nizhnaya Tunguska
River, $40 \mathrm{~km}$ upstream of the mouth, larch forest, litter\}, гарь, подстилка \{burnt forest, litter\}, 5.VIII.1978, А.А. Вахрушев \{A.A. Vakhrushev\}.

Ta-4194. Paratype +, Эвенкия, p. Кочечум (правый приток Нижней Тунгуски), 40 км выше устья \{Evenkiya, Kochechum River (right tributary of Nizhnaya Tunguska River, $40 \mathrm{~km}$ upstream of the mouth\}, гарь, подстилка \{burnt forest, litter\}, 5.VIII.1978, A.A. Вахрушев \{A.A. Vakhrushev\}.

Types are kept in ZMMU only.

Current status. Agyneta pseudosaxatilis Tanasevitch, 1984 (Linyphiidae).

ripariensis Tanasevitch, 1984, Agyneta (Aprolagus) Tanasevitch, 1984: 48-49, ðèñ. 2 (À- $\AA$ ).

Ta-4195. Holotype O', Полярный Урал (вост. макросклон), долина р. Собь, 20 км к западу от пос. Харп \{Polar Urals (eastern macroslope), Sob' River valley, $20 \mathrm{~km} \mathrm{~W}$ of Kharp\}, участок берега, занятый ерниковой (Betula nana) мохово-лишайниковой ассоциацией, подстилка \{river bank with dwarf birch (Betula nana) moss-lichen association\}, 19.VIII.1983, leg. А.В. Танасевич \{A.V. Tanasevitch\}.

Та-4196. Paratypes $2 \sigma^{7} \sigma^{7}, 5$ о⿱ , Коми АCCР, Воркутинский р-н, окр. пос. Мульда \{Komi Republic, Vorkuta Distr., nr. Mul'da\}, тундра, склон холма, кустарничковая мохово-лишайниковая ассоциация \{tundra, hill slope, shrubby moss-lichen association\}, 11.IX.1983, leg. A.В. Танасевич $\{$ A.V. Tanasevitch .

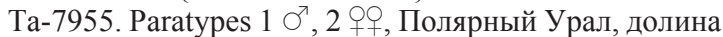
p. Собь, 20 км 3 от п. Харп, берег р. Собь \{Polar Urals, Sob' River valley, $20 \mathrm{~km} \mathrm{~W}$ of Kharp, Sob' River bank\}, подстилка $\{$ litter $\}, 19.8 .1983$, leg. А. $<$ В. $>$ Танасевич $\{$ A.V. Tanasevitch\}.

Other types are kept in ZIN.

Current status. Agyneta ripariensis Tanasevitch, 1984 (Linyphiidae).

\section{saaristoi Tanasevitch, 2000, Agyneta}

Tanasevitch, 2000: 206-209, figs 48-68.

Ta-7843. Paratype $\sigma^{7},<$ Kazakhstan $>$ КЗ [=КзылОрдинская обл.], Яныкурганский р-н, 35 км NNO г. Яныкурган, хр. Каратау \{Kzyl-Orda Area, Yanykurgan Distr., $35 \mathrm{~km}$ NNE of Yanykurgan, Karatau Mt. Ridge\}, 15.6. $<19>89<$, leg. A.A. Zyuzin $>$.

Other types are kept in ISEA, PSU and ZMUT.

The holotype and several paratypes are not yet transferred by the author to ZMMU.

Current status. Agyneta saaristoi Tanasevitch, 2000 (Linyphiidae).

sakhalinensis Saaristo et Marusik, 2004, Agyphantes

Saaristo, Marusik, 2004: 79, figs 7, 12-13, 19-21.

Ta-7240. Holotype $\sigma^{7}$, Sakhalin Island, central part, Leonidovka River (right tributary of Poronai River), $30 \mathrm{~km}$ downstream from Ulyanovka River mouth, $49^{\circ} 15.092^{\prime} \mathrm{N}$, $142^{\circ} 43.889^{\prime}$ E, 4-5 August 2001, Y.M. Marusik.

Ta-7241. Paratypes 2 ㅇ, 4 juv., Sakhalin Island, central part, Leonidovka River (right tributary of Poronai River), 30 $\mathrm{km}$ downstream from Ulyanovka River mouth, $49^{\circ} 15.092^{\prime} \mathrm{N}$, $142^{\circ} 43.889^{\prime} \mathrm{E}, 4-5$ August 2001, Y.M. Marusik.

Ta-7242. Paratype + , Sakhalin Island, central part, Leonidovka River (right tributary of Poronai River), $30 \mathrm{~km}$ downstream from Ulyanovka River mouth, $49^{\circ} 15.092^{\prime} \mathrm{N}$, $142^{\circ} 43.889^{\prime} \mathrm{E}, 4-5$ August 2001, Y.M. Marusik. 
Other types are kept in JW and ZMUT.

Current status. Agyphantes sakhalinensis Saaristo et Marusik, 2004 (Linyphiidae).

sayanensis Marusik, Azarkina et Koponen, 2004,

\section{Acantholycosa}

Marusik et al., 2004: 107-109, figs 10-14, map 1.

Ta-7545. Paratype $\sigma^{7}$, Krasnoyarsk Province, Yermakovski Distr., Abakan-Kyzyl Hwy $612^{\text {th }} \mathrm{km}$, Oiskoye Lake, 5.07.1989, D.L. Grodnitskiy.

The holotype is kept in ISEA.

Current status. Acantholycosa sayanensis Marusik, Azarkina et Koponen, 2004 (Lycosidae).

\section{serpentina Guo et Zhang, 2010, Aculepeira}

Guo, Zhang, 2010: 261-263, figs 1-5.

Ta-7700. Paratype + , Qiyi Farm $\left(29^{\circ} 38^{\prime} \mathrm{N}, 91^{\circ} 02^{\prime} \mathrm{E}\right)$, Lhasa City, Tibet Autonomous Region, 30 July 2002, Feng Zhang leg.

The holotype and other paratypes are kept in the Museum of Hebei University (Baoding, China).

Current status. Aculepeira serpentina Guo et Zhang, 2010 (Araneidae).

spirembolus Marusik, Azarkina et Koponen, 2004,

\section{Acantholycosa}

Marusik et al., 2004: 125-126, figs 143-146, map 1.

Ta-6776. Paratype $\sigma^{T}$, Altai, Kholzun Mt. Range, upper reaches of left tributary of Bannaya River, 2000-2250 m, mountain tundra, 13-14.06.1999, A. $<$ Yu. $>$ Dudko, R. $<$ Yu. $>$ Dudko.

The holotype is kept in ISEA.

Current status. Acantholycosa spirembolus Marusik, Azarkina et Koponen, 2004 (Lycosidae).

subnivalis Tanasevitch, 1989, Agyneta (Meioneta)

Tanasevitch, 1989: 91-92, figs 16-18.

Ta-5432. Holotype $\sigma^{7}$, Киргизия, Киргизский хр., пер. Тюя-Ашу \{Kirghizia, Kirghizsky Mt. Ridge, Tyuya-Ashu Pass \}, 3000-3400 м $\{\mathrm{m}\}$, 6-8.VIII.1984, собр. С.Л. Зонштейн \{coll. S.L. Zonstein\}.

Ta-5433. Paratype $\sigma^{7}$ [palp only], Киргизия, Центр. Тянь-шань, сырты, окр. метеост. “Тянь-Шань” \{Kirghizia, Central Tien-Shang, syrts, environs of Tien-Shang meteostation $\}, 3700-3900$ м $\{\mathrm{m}\}$, 15-25.vii.1983, собр. Б. Павлюк \{coll. B. Pavlyuk\}.

Ta-5434. Paratype ऽ", Киргизия, Центр. Тянь-шань, сырты, окр. метеост. "Тянь-Шань” \{Kirghizia, Central Tien-Shang, syrts, environs of Tien-Shang meteostation $\}$, 3700-3900 м $\{\mathrm{m}\}, 15-25 . v i i .1983$, собр. Б. Павлюк \{coll. B. Pavlyuk\}.

Ta-5435. Paratype $\sigma^{7},<$ Таджикистан, $>$ Туркестанский хребет, Кусавли-сай \{Tadjikistan, Turkestanskiy Mt. Ridge, Kusavli-Sai\}, арчевник, под камн $<$ ями $>$ \{Juniperus stand, under stones $\}, 2400-2700$ м $\{\mathrm{m}\}$, 29.IX.1970, собр. E.M. Андреева \{coll. Е.M. Andreeva\}.

Ta-7956. Paratypes 3 कо , <Киргизия,> Заалайский хр., сев. склон, Бордоба \{Kirghizia, Zaalayskiy Mt. Range, N slope, Bordoba $\}, 3500$ м $\{\mathrm{m}\}, 8 . \mathrm{X}-<19>70$, Е. $<$ М. $>$ Андреева $\{$ E.M. Andreeva $\}$.

Types are kept in ZMMU only.

Current status. Agyneta subnivalis Tanasevitch, 1989 (Linyphiidae). sundukovi Marusik, Azarkina et Koponen, 2004, Acantholycosa

Marusik et al., 2004: 128, figs 162-167, map 2.

Ta-7546. Holotype $\sigma^{7}$, Russia, Maritime Province, Lazo Reserve, Amerika kordon, $43^{\circ} 16^{\prime} \mathrm{N}, 134^{\circ} 03^{\prime} \mathrm{E}, 14-17.05$. 1999, Yu. $<$ N. $>$ Sundukov.

The species was described from the holotype only.

Current status. Acantholycosa sundukovi Marusik, Azarkina et Koponen, 2004 (Lycosidae).

talyshica Guseinov, Marusik et Koponen, 2005, Agelescape

Guseinov et al., 2005: 158-159, figs 13-16.

Ta-7519. Holotype + , SE Azerbaijan, Lerik Distr., env. of Lyalyakeran Vill., 1600 m, 23.08.1995, E.F. Guseinov.

Paratypes ( 7 + 9 ) have not yet been transferred to ZMMU.

Current status. Agelescape talyshica Guseinov, Marusik et Koponen, 2005 (Agelenidae). neta)

tianschanica Tanasevitch, 1989, Agyneta (MeioTanasevitch, 1989: 92, figs 6-11.

Ta-5436. Holotype $\sigma^{7}$, Киргизия, Сары-Челекский запов., Каратоко \{Kirghizia, Sary-Chelekskiy Reserve, Karatoko\}, сухой альп<ийский> луг, под камнями \{dry alpine meadow, under stones $\}, 3250$ м $\{\mathrm{m}\}$, 21.IX.1983, собр. А.Б. Рывкин \{coll. А.В. Ryvkin\}.

Тa-5437. Paratypes 1 ア , 2 фо, Киргизия, СарыЧелекский запов., Каратоко \{Kirghizia, Sary-Chelekskiy Reserve, Karatoko \}, сухой альп<ийский> луг, под камнями \{dry alpine meadow, under stones\}, 3250 м $\{\mathrm{m}\}, 21 . \mathrm{IX} .1983$, собр. А.Б. Рывкин \{coll. А.В. Ryvkin\}.

Та-5438. Paratypes $1 \sigma^{7}, 16$ 우 , Зап. Тянь-Шань, Ферганский хр., окр. пос. Арсланбоб \{W Tien-Shang, Ferghanskiy Mt. Range, environs of Arslanbob\}, сухой субальп <ийский> луг, под камн $<$ ями $>$ \{dry alpine meadow, under stones $\}, 2200$ м $\{\mathrm{m}\}, 1 . X .1983$, собр. К.Ю. Еськов \{coll. K.Yu. Eskov\}.

Ta-5439. Paratype + [epigyne only], Зап. Тянь-Шань, Ферганский хр., окр. пос. Арсланбоб \{W Tien-Shang, Ferghanskiy Mt. Range, environs of Arslanbob $\}$, сухой субальп<ийский> луг, под камн<ями $>$ \{dry alpine meadow, under stones $\}, 2200$ м $\{\mathrm{m}\}, 1$. Х.1983, собр. К.Ю. Еськов \{coll. K.Yu. Eskov\}.

Other types are kept in SMF.

Current status. Agyneta tianschanica Tanasevitch, 1989 (Linyphiidae).

\section{tibialis Tanasevitch, 2005, Agyneta}

Tanasevitch, 2005: 165-166, figs 1-8.

Ta-7827. Holotype $\sigma^{7}$, Алтай, Загриха \{Altais, Zagrikha\}, 1700 м $\{\mathrm{m}\}, 27.6 .[20] 00, \Gamma .<\mathrm{H} .>$ Азаркина \{G.N. Azarkina\}.

The types should be kept in ZMMU only; one paratype $\left(O^{7}\right)$ should still be transferred to ZMMU.

Current status. Agyneta tibialis Tanasevitch, 2005 (Linyphiidae).

uzbekistanica Tanasevitch, 1984, Agyneta (Meioneta)

Tanasevitch, 1984: 51-52, ðèñ. 5 (À-Ç).

Ta-4202. Holotype O?, Узбекская CCP, Ташкентская область, Бостанлыкский p-н, близ поселка Сиджак \{Usbek SSR, Tashkent Area, Bostanlyk Distr., near Sidzhak\}, склон горы (2000 м над уровнем моря), арчевое ред- 
колесье, под камнями \{mountain slope (2000 m a.s.1.), Juniperus sparse stand, under stones $\}$, 10.IV.1982, leg. A.B. Танасевич $\{$ A.V. Tanasevitch $\}$.

Other types are kept in ZIN.

Current status. Agyneta uzbekistanica Tanasevitch, 1984 (Linyphiidae).

\section{variegatus Kroneberg, 1875, Aelurops}

Kroneberg, 1875: 51, tab. V, fig. 39.

Ta-1049. Syntype + , Туркестанская Ученая Экспедиция Императорского Общества Любителей Естествознания. <А.П.> Федченко \{Turkestan Scientific Expedition by the Imperator Society for Friends of Natural Sciences. A.P. Fedchenko\}. 16. Самарканд \{Samarkand\}.

Ta-1050. Syntypes $1 \sigma^{7}$ [with the palp detached], 1 , Туркестанская Ученая Экспедиция Императорского Общества Любителей Естествознания. <А.П.> Федченко \{Turkestan Scientific Expedition by the Imperator Society for Friends of Natural Sciences. A.P. Fedchenko\}. 15. Ходжадук \{Khodzhaduk\}.

Types are kept in ZMMU only.

Current status. Rafalus variegatus (Kroneberg, 1875) (Salticidae).

yakutsaxatilis Marusik et Koponen in Marusik, Koponen, Vinokurov et Nogovitsyna, 2002, Agyneta

Marusik et al., 2002: 357, figs 19-23, 31-36, 40-42, 46-47.

Ta-6538. Holotype $\sigma^{7}$, Russia, North Yakutia, Yana River down flow, Kular Vill. env., $70.35^{\circ} \mathrm{N}, 134.34^{\circ} \mathrm{E}$, July 1996 , leg. N.N. Vinokurov.

Ta-6539. Paratypes $2 \sigma^{7} \sigma^{7}, 1$, Russia, North Yakutia, Yana River down flow, Kular Vill. env., $70.35^{\circ} \mathrm{N}, 134.34^{\circ} \mathrm{E}$, July 1996, leg. N.N. Vinokurov.

Other types are kept in JW and ZMUT.

Current status. Synonymized with Agyneta amersaxatilis Saaristo et Koponen, 1998 (Linyphiidae) [Tanasevitch, 2010].

zinchenkoi Marusik, Azarkina et Koponen, 2004,

\section{Acantholycosa}

Marusik et al., 2004: 123-124, figs 57, 135-142, map 1 .

Ta-6778. Paratype + , Altai, South Altai, south part of Katun Mt. Range, 5 km south-east of Rakhmanovskiye Klyuchi (=springs), 2100-2500 m, alpine zone, 28.06.1997, $\mathrm{R} .<\mathrm{Yu}$. $>$ Dudko, V. $<\mathrm{K} .>$ Zinchenko.

Other types are kept in ISEA (holotype, paratypes), JW (now in SMF) and ZMUT.

The type locality is situated in East Kazakhstan Area (Kazakhstan) rather than in Russia, as stated in the original description.

Current status. Acantholycosa zinchenkoi Marusik, Azarkina et Koponen, 2004 (Lycosidae).

zonsteini Marusik et Omelko, 2017, Acantholy$\cos a$

Marusik, Omelko, 2017: 597-599, figs 1-9.

Ta-8006. Holotype O', Khabarovsk Prov., Slavyanka, 1990, S. $<$ I. $>$ Golovatch.

The species was described from the holotype only.

Current status. Acantholycosa zonsteini Marusik et Omelko, 2017 (Lycosidae).

Acknowledgements. I am cordially obliged to my colleague Dmitry V. Logunov (Manchester, UK) for reviewing the manuscript and linguistic help. Comments from Galina
G. Krivosheina (Moscow, Russia) are highly appreciated. This paper is supported by the Russian Science Foundation Project No. 14-50-00029. The curatorial work with museum registers and other paper documentation (card indexes, etc.) was supported by the Moscow State University Project No. AAAA-A16-116021660077-3

\section{References}

Azarkina G.N. 2002. New and poorly known species of the genus Aelurillus Simon, 1884 from Central Asia, Asia Minor and the eastern Mediterranean // Bull. Brit. arachnol. Soc. Vol.12. Pt.6. P.249-263.

Azarkina G.N. 2003. Aelurillus ater (Kroneberg, 1875) and related species of jumping spiders in the fauna of Middle Asia and the Caucasus (Aranel: Salticidae) // Arthropoda Selecta. Vol.11(for 2002). No.1. P.89-107.

Azarkina G.N., Mirshamsi O. 2014. Description of a new Aelurillus species from Khorasan province of Iran, with comments on A. concolor Kulczyński, 1901 (Araneae: Salticidae) // Zoology in the Middle East. Vol.60. No.1. P.82-91.

Dunin P.M. 1984. [Fauna and ecology of spiders (Aranei) of the Apsheron Peninsula (Azerbaijanian SSR)] // Utochkin A.S. (ed.). Fauna i ekologiya paukoobraznykh. Perm: Perm Univ. P.45-60 [in Russian].

Esyunin S.L. 2017. New data on Aituaria pontica (Spassky, 1932) (Aranei: Nesticidae) // Arthropoda Selecta. Vol.26. No.3. P.241243.

Esyunin S.L., Efimik V.E. 1998. Remarks on the Ural spider fauna, 8. New and unidentified species from steppe landscapes of the South Urals (Arachnida: Aranei) // Arthropoda Selecta. Vol.7. No.2. P.145-152.

Fomichev A.A., Marusik Yu.M., Koponen S. 2016. On the synonymy of two Acantholycosa species (Araneae, Lycosidae) from the Altai // ZooKeys. Vol.559. P.151-156.

Guo Sheng-Tao, Zhang Feng. 2010. A new species of the genus Aculepeira from Qinghai-Tibet Plateau, China (Aranei: Araneidae) // Arthropoda Selecta. Vol.19. No.4. P.261-263.

Guseinov E.F., Marusik Yu.M., Koponen S. 2005. Spiders (Arachnida, Aranei) of Azerbaijan. 5. Faunistic review of the funnelweb spiders (Agelenidae) with description of new genus and species // Arthropoda Selecta. Vol.14. No.2. P.153-177.

Kroneberg A.I. 1875. [A.P. Fedchenko. Voyage in Turkestan. Spiders. Araneae] // Izvestiya Obshchestva lyubitelei estestvoznaniya, antropologii i etnografii. Vol.19. No.3. P.I-IV, 1-55, 5 tabs [in Russian].

Logunov D.V. 1993. New data on the jumping spiders (Araneae Salticidae) of Mongolia and Tuva // Arthropoda Selecta. Vol.2. No.2. P.47-53.

Logunov D.V., Marusik Yu.M. 2000. Miscellaneous notes on Palaearctic Salticidae (Arachnida: Aranei) // Arthropoda Selecta. Vol.8 (for 1999). No.4. P.263-292.

Marusik Yu.M., Azarkina G.N., Koponen S. 2004. A survey of East Palaearctic Lycosidae (Aranei). II. Genus Acantholycosa and related new genera // Arthropoda Selecta. Vol.12 (for 2003). No.2. P.101-148.

Marusik Yu.M., Koponen S., Vinokurov N.N., Nogovitsyna S.N. 2002. Spiders (Aranei) from the northernmost forest-tundra of northeastern Yakutia $\left(70^{\circ} 35^{\prime} \mathrm{N}, 134^{\circ} 34^{\prime}\right.$ E) with description of three new species // Arthropoda Selecta. Vol.10 (for 2001). No.4. P.351-370.

Marusik Yu.M., Omelko M.M. 2011. A survey of East Palaearctic Lycosidae (Araneae). 7. A new species of Acantholycosa Dahl, 1908 from the Russian Far East // ZooKeys. Vol.79. P.1-10.

Marusik Yu.M., Omelko M.M. 2017. A new species of Acantholycosa baltoroi-group (Araneae: Lycosidae, Pardosinae) from the Russian Far East // Zootaxa. Vol.4232. No.4. P.597-600.

Mikhailov K.G. 2016a. Arachnid types in the Zoological Museum, Moscow State University. I. Opiliones (Arachnida) // Arthropoda Selecta. Vol.25. No.3. P.327-334. 
Mikhailov K.G. 2016b. [Arachnology in Russia/USSR] // Pavlinov I.Ya., Kalyakin M.V., Sysoev A.V. (eds.). Aspects of biodiversity. Part 2. Archives of Zoological Museum of Moscow State University. Vol.54. No.2. P.655-691 [in Russian, with English summary]

Ponomarev A.V. 2007. [New taxa of spiders (Aranei) from the south of Russia and West Kazakhstan] // Caucasian Entomol. Bull. Vol.3. No.2. P.87-95 [in Russian, with English summary].

Saaristo M., Marusik Yu.M. 2004. Two new petrophilous micronetine genera, Agyphantes gen.n and Lidia gen.n. (Araneae, Linyphiidae, Micronetinae) from the eastern Palaearctic with descriptions of two new species // Bull. Brit. arachnol. Soc. Vol.13. Pt.3. P.76-82.

Tanasevitch A.V. 1984. [New species of spiders of the genus Agyneta Hull, 1911 (Aranei, Linyphiidae) from Siberia and Middle Asia] // Nauchnye doklady vysshei shkoly. Biologicheskir nauki. No.5. P.47-53 [in Russian, with English summary].

Tanasevitch A.V. 1989. The linyphiid spiders of Middle Asia (Arachnida: Araneae: Linyphiidae) // Senckenberg. biol. Bd.69. H.1/3. P.83-176.

Tanasevitch A.V. 2000. On some Palaearctic species of the spider genus Agyneta Hull, 1911, with description of four new species (Aranei: Linyphiidae) // Arthropoda Selecta. Vol.8 (for 1999). No.3. P.201-213.
Tanasevitch A.V. 2005. New or little-known species of Agyneta and Nippononeta from Asia (Aranei: Linyphiidae) // Arthropoda Selecta. Vol.13 (for 2004). No.3. P.165-170.

Tanasevitch A.V. 2006. Five new Linyphiidae spiders from the Russian Far East, with notes on synonymy (Arachnida: Aranei) // Arthropoda Selecta. Vol.15. No.1. P.29-38.

Tanasevitch A.V. 2010. On synonymy of linyphiid spiders of the Russian fauna (Arachnida: Aranei: Linyphiidae). 1 // Arthropoda Selecta. Vol.19. No.4. P.273-282.

Tanasevitch A.V. 2014. Two new Agyneta Hull, 1911 from the Asian part of Russia (Aranei: Linyphiidae) // Arthropoda Selecta. Vol.23. No.1. P.77-80.

Tanasevitch A.V. 2016. A new Agyneta Hull, 1911 from northern Yakutia, Russia (Aranei: Linyphiidae) // Arthropoda Selecta. Vol.25. No.1. P.75-76.

Wesołowska W. 1996. New data on the jumping spiders of Turkmenistan (Aranei Salticidae) // Arthropoda Selecta. Vol.5. Nos 1-2. P.17-53.

WSC. 2018. World Spider Catalog. Natural History Museum Bern, online at: http://wsc.nmbe.ch, version 19.0 (accessed on March 3rd, 2018).

Zyuzin A.A., Marusik Yu.M. 1988. [A new spider species of the genus Acantholycosa (Aranei, Lycosidae) from East Siberia] // Zool. Zhurn. Vol.67. No.7. P.1083-1085 [in Russian, with Englich summary].

Responsible editor D.V. Logunov 